\title{
Guanine Nucleotide Exchange Factor $7 B$ (RopGEF7B) is involved in floral organ development in Oryza sativa
}

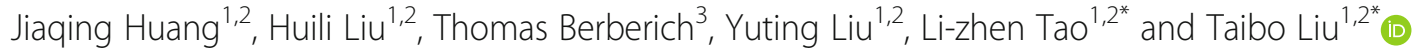

\begin{abstract}
Background: RAC/ROP GTPase are versatile signaling molecules controlling diverse biological processes including cell polarity establishment, cell growth, morphogenesis, hormone responses and many other cellular processes in plants. The activities of ROPs are positively regulated by guanine nucleotide exchange factors (GEFs). Evidence suggests that RopGEFs regulate polar auxin transport and polar growth in pollen tube in Arabidopsis thaliana. However, the biological functions of rice RopGEFs during plant development remain largely unknown.

Results: We investigated a member of the OsRopGEF family, namely OsRopGEF7B. OsRopGEF7B pro:GUS analysis indicates that OsRopGEF7B is expressed in various tissues, especially in the floral meristem and floral organ primordia. Knock-out and -down of OsRopGEFTB by T-DNA insertion and RNA interference, respectively, predominantly caused an increase in the number of floral organs in the inner whorls (stamen and ovary), as well as abnormal paleae/lemmas and ectopic growth of lodicules, resulting in decline of rice seed setting. Bimolecular fluorescence complement (BiFC) assays as well as yeast two-hybrid assays indicate that OsRopGEF7B interacts with OsRACs.
\end{abstract}

Conclusions: OsRopGEFTB plays roles in floral organ development in rice, affecting rice seed setting rate. Manipulation of OsRopGEF7B has potential for application in genetically modified crops.

Keywords: Agronomic traits, Floral development, OsRAC, OsRopGEF, Rice

\section{Background}

RAC/ROPs, plant Rho-like small G proteins, are multi-functional signaling switches regulating many cellular processes in plants, affecting leaf epidermal cell morphogenesis, polarized cell growth in pollen tubes and root hairs, and hormone and defense-related responses (Yang and Fu 2007; Yalovsky et al. 2008; Wu et al. 2011; Nibau et al. 2013; Huang et al. 2014). RAC/ ROPs are essential signaling molecules that switch between a GTP-bound active form and a GDP-bound inactive form (Bourne et al. 1990, 1991; Wu et al. 2011). Activation of GTPases depends on guanine nucleotide exchange factors (GEFs) that stimulate the exchange of GDP- to GTP-bound form (Cherfils and Chardin 1999; Shichrur and Yalovsky 2006; Wu et al. 2011). It was

\footnotetext{
* Correspondence: Iztao2005@scau.edu.cn; tbliu@scau.edu.cn

${ }^{1}$ State Key Laboratory for Conservation and Utilization of Subtropical Agro-bioresources, South China Agricultural University, Guangzhou 510642, China

Full list of author information is available at the end of the article
}

shown that RAC/ROPs utilize mostly a plant-specific family of GEFs named RopGEFs for activation in plant kingdom (Berken et al. 2005).

Arabidopsis contains 14 RopGEFs in its genome, sequentially termed RopGEF1 to RopGEF14, sharing a conserved PRONE domain for GEF catalytic activity. Transient expression analyses provide evidence that RopGEF1 and RopGEF12 regulate polarized pollen tube growth (Gu et al. 2006; Zhang and McCormick 2007). ABA-mediated degradation of RopGEF1 also plays an important role in ABA-mediated inhibition of lateral root growth (Li et al. 2016). Most recently, our group reported that RopGEF1 is very important in the plant early development via affecting cell polarity and polar auxin transport (Liu et al. 2017). The study of a ropgef1ropgef4 double mutant suggests that RopGEF1 and RopGEF4 are specific regulators of ROP11 function in ABA-mediated stomatal closure (Li and Liu 2012). Arabidopsis full-ge nome chip transcriptome assay results in combination with physiological studies further support that 
RopGEF10 negatively regulates ABA responses by inducing a particular subset of genes associated with stress responses (Xin et al. 2005). Previously, our group has reported that Arabidopsis RopGEF7, is specifically expressed in the quiescent center (QC) precursors during embryogenesis and in the QC of postembryonic roots, regulating PLT-mediated maintenance of root meristem by connecting RopGEF-regulated RAC/ROP signaling and polar auxin transport (Chen et al. 2011). Furthermore, ROP3 interacts directly with RopGEF7 (Chen et al. 2011) and regulates Arabidopsis embryo development and seedling growth via affecting the polar auxin transport and thus controlling the establishment of auxin maxima (Huang et al. 2014).

Rice contains 11 RopGEFs in its genome (Berken et al. 2005; Gu et al. 2006; Yoo et al. 2011). It was shown that PRONE-type RacGEFs in rice may play a role in the activation of OsRac1 in disease resistance response (Kawasaki et al. 2009). The expression levels of some OsRacGEFs were affected by treatment with sphingolipid elicitors (SE), implying that some OsRacGEFs may be regulated at the transcription level during plant response to stress (Kawasaki et al. 2009). OsRopGEF10 predominantly expressed in newly developed leaves before the appearance from the leaf sheath and regulated small papillae development (Yoo et al. 2011). However, studies on RopGEFs in rice are still limited.

In this work, we examined a RopGEF member in rice, namely OsRopGEF7B, which is a homolog of AtRopGEF7. AtRopGEF7 was reported to be essential for root meristem maintenance (Chen et al. 2011). Our results indicated that OsRopGEF7B was highly expressed in root meristem, floral meristem, floral organ primordia, and the inner floral organs. We further explored the roles of $O s R o p G E F 7 B$ in rice development by analyzing the osropgef $7 b-1$ mutant and OsRopGEF7B-RNAi plants, both of which displayed variedly defective phenotypes, including increased number of stamens and ovaries, abnormal palea/lemma, and shortened plant height. Taken together, these results implicate that OsRopGEF7B plays a role in regulating floral organ development, thus subsequently affects rice seed setting rate.

\section{Results}

\section{OsRopGEF7B is Expressed in Various Tissues,}

\section{Predominantly in Floral Meristem and Floral Organs}

To examine the expression pattern of OsRopGEF7B, we carried out quantitative RT-PCR (qRT-PCR) and promot er-GUS reporter gene analysis in various tissues at seedling and floral stages. qRT-PCR analysis showed that OsRopGEF7B was expressed in roots, stems, flag leaves, flowers, and immature seeds, specifically in flowers (Additional file 1: Figure S1). To further verify these observations, we analyzed the GUS ( $\beta$-glucuronidase) activity in several OsRopGEF7B $B_{\text {pro }}$ GUS transgenic lines.
OsRopGEF7B was expressed in the root of 5-day-old seedling (Fig. 1a), especially in the meristematic region of root tip (Fig. 1b). GUS activities were also detected in lateral root primordia and lateral root (Fig. 1c), and vein of the third leaf (Fig. 1d) at seedling stages. The OsRop$G E F 7 B$ expression was also observed in the floral meristem and floral organ primordia of different stages in the transgenic plants (Fig. 1e-h), predominantly in the later-stage Sp4 (Fig. 1g) and Sp6 (Fig. 1h). When the plants reached reproductive stages, GUS activity was high in anthers, filaments and stigmas (Fig. 1i-1), most predominantly in pollen (Fig. 11). These results are in accordance with the microarray data from the RiceXPro online databases (http://ricexpro.dna.affrc.go.jp/GGEP/ graph-view.php?featurenum=3833).

\section{Loss-of-Function Mutation of OsRopGEF7B Causes Developmental Defects in Floral Organs}

A T-DNA insertion line (named osropgef7b-1) was identified. T-DNA insertion was confirmed in the seventh exon (PFG_3A-10,465) of OsRopGEF7B (Fig. 2a) by PCR analysis. It was confirmed as a knock out mutant as qRT-PCR assay displayed absence of OsRopGEF7B transcript in this mutant (Fig. 2b). Since OsRopGEF7B was highly expressed in floral meristem and floral organs (Fig. 1e-l), we focused our analysis on floral development. Phenotype analysis indicated that loss of function in OsRopGEF7B severely affects flower organ development, $18.6 \%$ flowers (195 in 1046 flowers) showed defects (Table 1; Fig. 2f-r). In detail, the OsRopGEF7B mutant exhibited phenotype of a flower with an abnormal palea/lemma, an elongated sterile lemma, or homeotic transformation of a sterile lemma into a lemma structure $(2.58 \%, n=1046$; Table 1 ; Fig. 2f-h), compared with wild type (Table 1; Fig. 2c-e). A flower has two ovaries $(10.13 \%, n=1046$; Table 1; Fig. $2 \mathrm{k}$, $\mathrm{n}, \mathrm{p})$ or more than two ovaries $(1.34 \%, n=1046$; Table 1 ; Fig. 2i-j, l-m, o, q) in osropgef $7 b-1$ mutant, while wild type normally contains only one ovary in a flower (Fig. 2c-e). A flower with multiple ovaries always contains more than six stamens (11.7\%, n = 1046; Table 1; Fig. 2k). Additionally, we observed two flowers shared with one receptacle $(0.57 \%, n=1046$; Table 1; Fig. 2r). Transverse section of spikelets of osropgef $7 b-1$ displayed that an osropgef $7 b-1$ flower contains ten stamens (Fig. 3b), and the maximum could be up to 12 stamens (Fig. 3c, d), while wild type only contains six stamens (Fig. 3a). The corresponding results of transverse section of spikelets (Fig. 3a-d) were illustrated more clearly by using the diagrams (Fig. 3e-h), respectively.

\section{Knockdown of OsRopGEF7B Induces the Similar Floral Phenotypes to Those of the T-DNA Insertion Line} The osropgef $7 b-1$ showed defects in flower development (Table 1; Fig. 2f-r). To further confirm the role of 


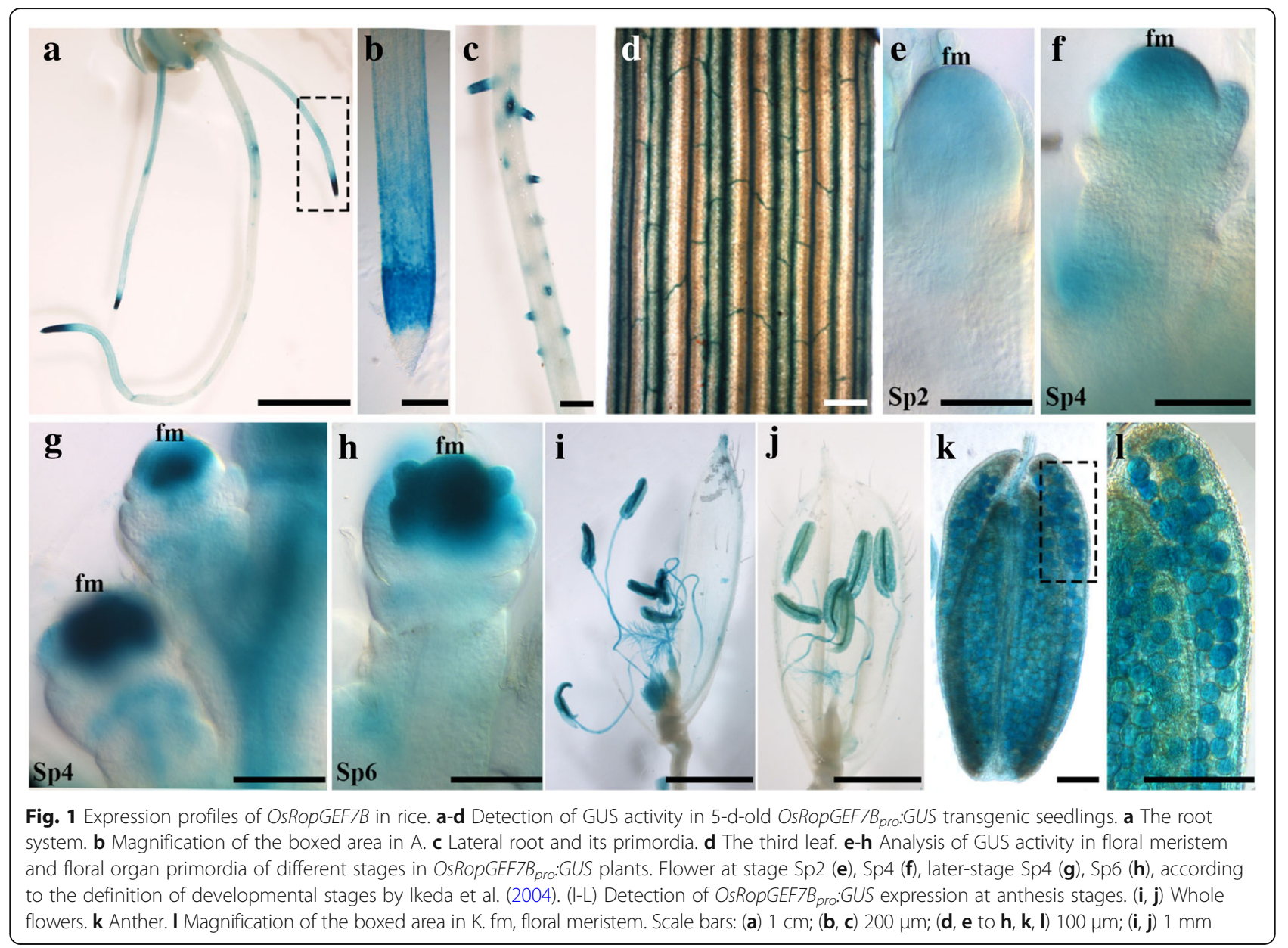

OsRopGEF7B, we generated the OsRopGEF7B-RNAi knockdown lines. In total, we obtained $40 \mathrm{~T} 1$ independent OsRopGEF7B-RNAi lines, and examined 11 lines by qRT-PCR (Additional file 1: Figure S2). The RNAi L31 with the strongest suppression in OsRopGEF7B mRNA levels (Fig. 4a) was chosen for subsequent analysis.

RNAi L31 showed very similar floral phenotypes as osropgef $7 b-1$, in detail, nearly 8\% flowers (106 in 1366 flowers) observed displayed developmental defects, including abnormal palea / lemma or elongated sterile lemma $(0.81 \%, n=1366$; Table 1 ; Fig. $4 \mathrm{e})$, more than six stamens (4.83\%, $n=1366$; Table 1; Fig. 4f), two ovaries (3.95\%, $n=1366$; Table 1; Fig. 4g, j), more than two ovaries $(0.88 \%, \mathrm{n}=1366$; Table 1 ; Fig. $4 \mathrm{~h})$, and multiple stigmas $(1.68 \%, n=1366$; Table 1; Fig. 4i). The lower percentage of developmental defects of RNAi L31 in flower compared with osropgef $7 b-1$ could be explained by the fact that the transcript level of OsRopGEF7B in RNAi L31 still remains over 20\% (Fig. 4a), but could not be detected by qRT-PCR in osropgef7b-1 (Fig. 2b). Thus, we proposed that OsRopGEF7B plays roles in floral organ development.

\section{Knock-off and -down of OsRopGEF7B Affects Agronomic Traits in Rice}

Since the osropgef $7 b-1$ mutant and RNAi L31 transgenic plant displayed similar floral phenotypes (Table 1; Figs. 2f-r, 4e-j), we further analyzed their agronomic traits. Plant height of both, osropgef7b-1 and RNAi L31, was markedly reduced in 7-day-old seedlings (Fig. 5a,d) and even in mature stage (Fig. 5b, e, g), compared with wild type. Panicles length and seed setting rate were notably reduced in both osropgef $7 b-1$ and RNAi L31 relative to the control (Fig. 5c, f, h, i). In addition, both of the first branch length (Fig. 5j) and the numbers of total grain per panicle (Fig. 5k) slightly decreased, compared with their controls.

Because OsRopGEF7B was highly expressed in pollen (Fig. 1k, l), it likely plays roles in pollen development similar to the homolog in Arabidopsis, AtRopGEF12. Overexpression of a C-terminally truncated AtRopGRF12 interrupted pollen tube growth (Zhang and McCormick 2007). Thereofore, we analyzed the pollen development of osropgef $7 b-1$ and RNAi L31, and our observations indicated that the percentage of pollen fertility from osropgef $7 b-1$ mutant and 

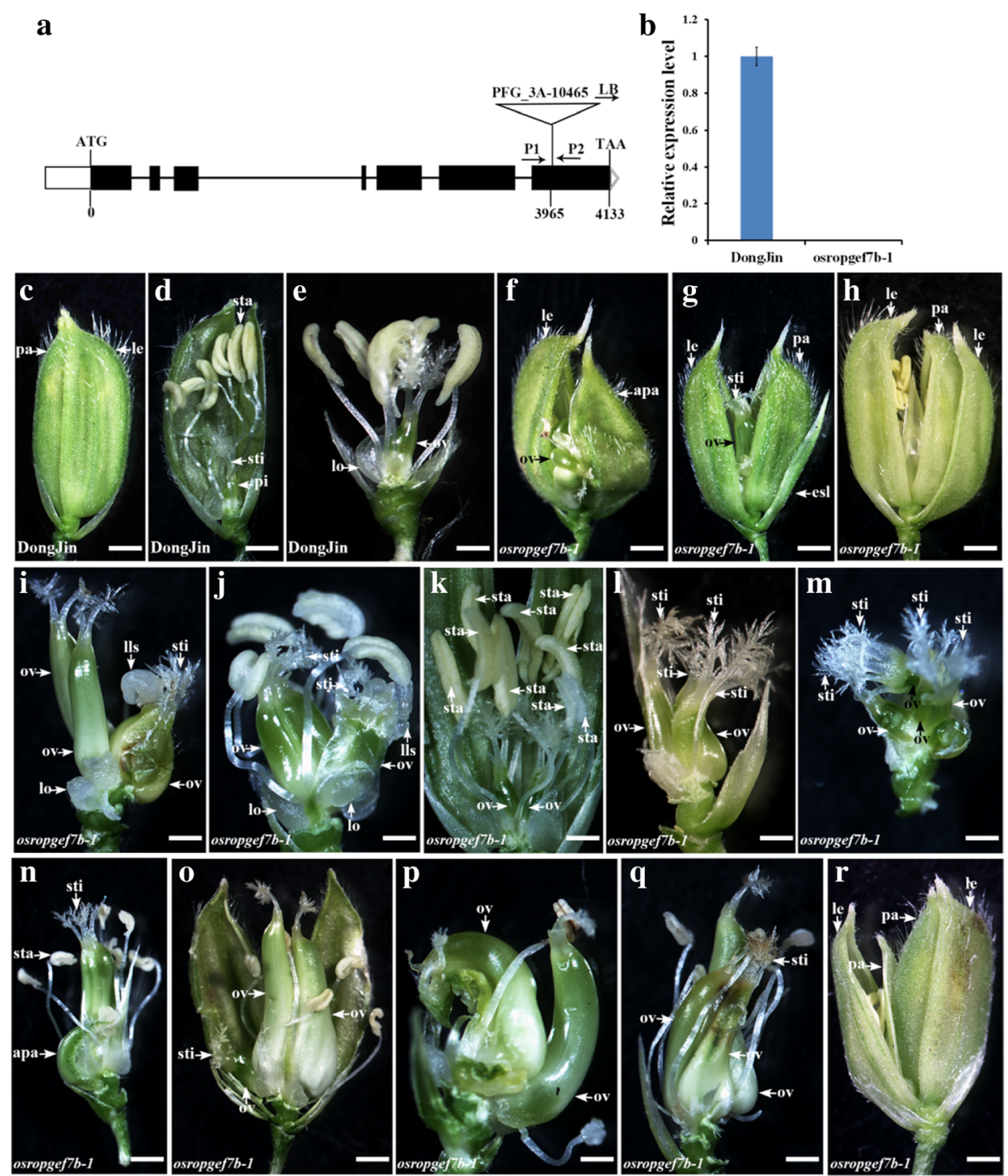

Fig. 2 Knock-out of OsRopGEFTB induces severe developmental defects in floral organ. a Structure of OsRopGEF7B. Black boxes indicate exons, black thin lines between exons indicate introns, white boxes indicate UTRs. The T-DNA insertion site in the seventh exon of osropgef7b-1(PFG_3A10,465) is shown and P1 and P2 indicate the sites of primers used for identifying the T-DNA insertion. b Transcript level of OsRopGEF7B in osropgef7b-1 compared with wild type (DongJin). c-r Floral phenotypes of osropgef7b-1 compared with wild type. Lemmas/paleae and stamens were partially or totally removed to display the inner organs. c-e) Wild type flowers. $\mathbf{f}-\mathbf{r}$ ) osropgef $7 b-1$ flowers with different phenotypes. $\mathbf{f}$ A flower with one normal lemma and one abnormal palea, and an enlarged ovary coming out from the shells (lemma/palea). $\mathbf{g}$ A flower with an elongated sterile lemma. $\mathbf{h}$ A sterile lemma transformed into a lemma structure. $\mathbf{i}$-j Flowers have multiple ovaries with lodicules-like structure ectopic growth upon the top of ovary. (K) A flower with two ovaries and nine stamens. I-m) Flowers with multiple ovaries and stigmas. (n-q) Flowers with multiple fused or separated ovaries. $\mathbf{r}$ Two flowers in different sizes on a receptacle. Apa, abnormal palea; esl, elongated sterile lemma; glo, glume-like organ; le, lemma; Ils, lodicules-like structure; lo, lodicules; ov, ovary; pa, palea; pi, pistil; sta, stamen; sti, stigma; Scale bars: (c to r) $1 \mathrm{~mm}$

RNAi L31 plant (Additional file 1: Figure S3B, D, I) are same to those of the wild type controls (Additional file 1: Figure S3A, C, I). Results of DAPI staining showed that all the fertile pollen grains from osropgef7b-1, RNAi L31 and the wild type contained three nuclei: two bright, intensely stained sperm nuclei and one diffused, weakly stained vegetative nucleus (Additional file 1: Figure S3E-H). The DAPI analysis indicated that the knock-out or -down of OsRopGEF7B did not affect pollen development. We also found that pollen tube elongation and pollen germination in vivo of both osropgef7b-1 mutant (Additional file 1: Figure S4D-F, M) and RNAi L31 plant (Additional file 1: Figure S4J-M) were not different from those of the wild type (Additional file 1: Figure S4A-C, G-I, M), indicating that the knock-out or -down of OsRopGEF7B did not affect pollen tube elongation. Taken together, our data suggest that the reduced seed setting of osropgef $7 b-1$ mutant and RNAi plants might be partly attributable to the defects in female organ development, as pollen development and pollen tube growth in osropgef7b-1 and RNAi plants are quite normal. 
Table 1 Numbers of floral organs in wild-type and osropgef7b-1 plants

\begin{tabular}{llllllll}
\hline & $\begin{array}{l}\text { Two } \\
\text { ovaries }\end{array}$ & $\begin{array}{l}\text { More than two } \\
\text { ovaries }\end{array}$ & $\begin{array}{l}\text { Multiple } \\
\text { stigmas }\end{array}$ & $\begin{array}{l}\text { Abnormal paleae/ } \\
\text { lemmas }\end{array}$ & $\begin{array}{l}\text { Two } \\
\text { flowers }\end{array}$ & $\begin{array}{l}\text { Number of stamens } \\
(n>6)\end{array}$ & $\begin{array}{l}\text { Total flowers } \\
\text { analyzed }\end{array}$ \\
\hline $\begin{array}{l}\text { DongJin } \\
\text { osropgef7b- }\end{array}$ & $\begin{array}{l}106 \\
1\end{array}$ & 0 & 0 & 0 & 0 & 0 & 1151 \\
ZH11 & 0 & $14(1.34 \%)$ & $42(4.02 \%)$ & $27(2.58 \%)$ & $6(0.57 \%)$ & $120(11.7 \%)$ & 1046 \\
RNAi L31 & $54(3.95 \%)$ & $12(0.88 \%)$ & $23(1.68 \%)$ & $11(0.81 \%)$ & 0 & 0 & 1420 \\
\hline
\end{tabular}

Multiple stigmas, flower with three or more than three stigmas were counted as flower containing multiple stigmas. Flower with two or more than two ovaries always contains more than six stamens. Data were presented as mean values of three biological repeats, $n=1009$ to 1420

OsRopGEF7B Does Not Affect the Expression of a Set of Transcription Factors Which are Associated with Floral Development in Rice

To further investigate how OsRopGEF7B works in regulating flower development, we examined several genes which encode transcription factors associated with floral development. Genetic studies showed that mutation in $Y A B B Y_{S}, E T T I N$, OsMADS1, OsMADS6 and OsMADS55 affect floral organ identification (Sessions et al. 1997; Nemhauser et al. 2000; Prasad et al. 2005; Yadav et al. 2011; Teo et al. 2014). Therefore, we carried out qRT-PCR to analyze the transcripts of these genes in

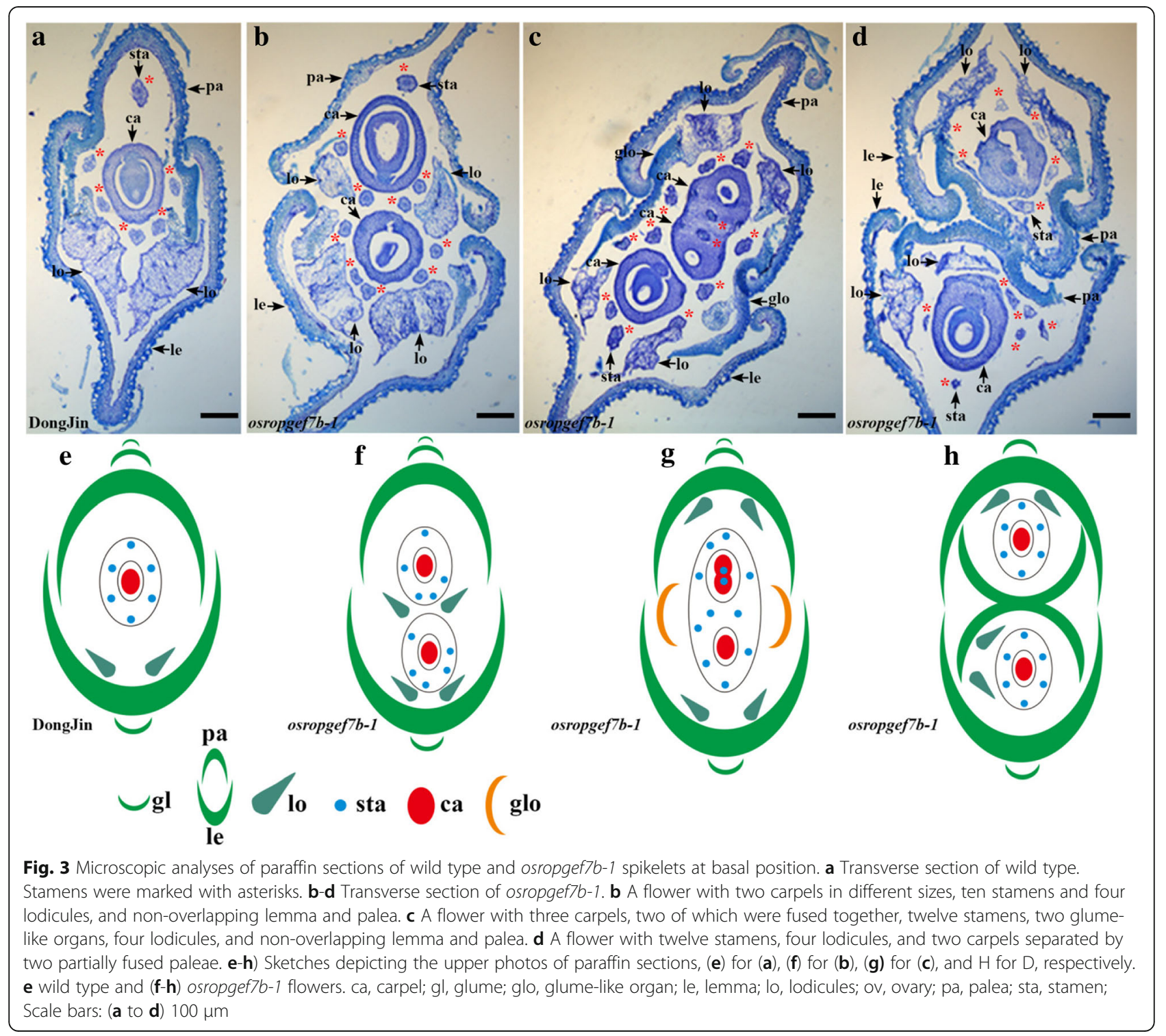



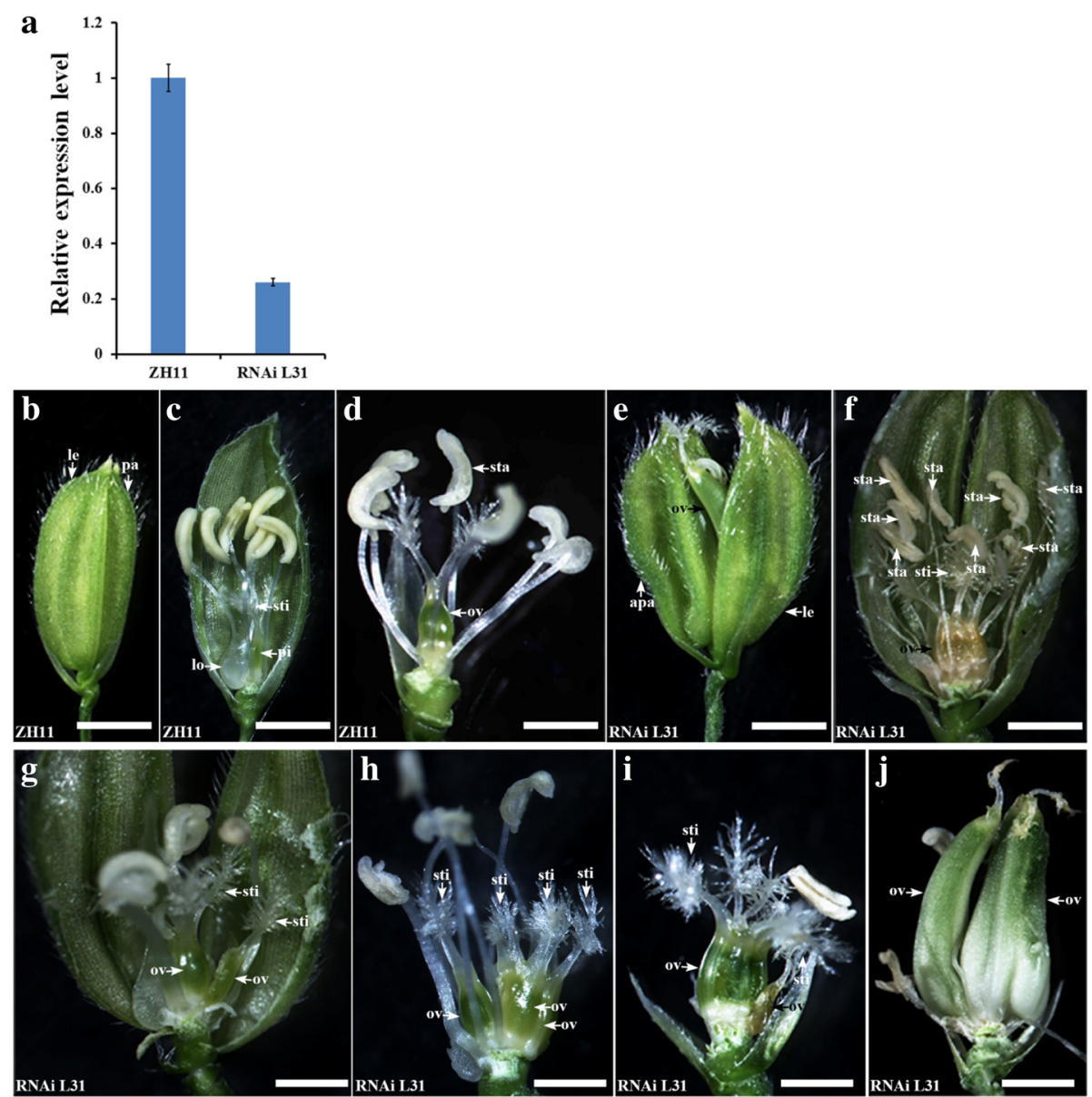

Fig. 4 Phenotypes of flowers in RNAi L31 transgenic plants. a Expression levels of OsRopGEF7B in RNAi L31 compared with wild type (ZH11). (b-j) Floral phenotypes of RNAi L31 compared with wild type. Lemmas/paleae and stamens were partially or totally removed to show the inner organs. b-d Wild type flowers. e-j RNAi L31 flowers with various phenotypes. e A flower with one normal lemma and one abnormal palea, and the ovary coming out from the shells. $\mathbf{f}$ A flower with eight stamens. $\mathbf{g}$ A flower with two ovaries. $\mathbf{g}$ A flower with more than two ovaries (i) A flower with multiple stigmas. $\mathbf{j}$ A flower with two enlarged different sized ovaries with atrophic stigmas. Bars $=1 \mathrm{~mm}$

both osropgef7b-1 and RNAi L31 at both seedling and floral stages. The results indicated that the genes analyzed were not significantly affected by OsRopGEF7B mutation (Additional file 1: Figure S5A, B).

We previously reported that knock-down of RopGEF7 affects PIN1 accumulation and polarization to impact polar auxin transport and thereby influences embryo and root development in Arabidopsis (Chen et al. 2011). PIN1 is also required for floral development (Yamaguchi et al. 2013; Holt et al. 2014). Thus we performed the qRT-PCR analysis of four OsPINs, and the data showed no changes in neither of them compared with wild type at both seedling and floral stages (Additional file 1: Figure S5C, D). Our data suggest that OsRopGEF7B might do not influence the expression of YABBYs, ETTIN, OsMADS1, OsMADS6, OsMADS55 and PIN1 in rice.

\section{OsRopGEF7B interacts with OsRACs in Rice Protoplasts} and Yeast Cells

Biochemical, structural and functional studies suggest that Arabidopsis RopGEFs act as activators of ROP/ RACs (Berken et al. 2005; Thomas et al. 2007; Craddock et al. 2012; Bloch and Yalovsky 2013; Chang et al. 2013; Yalovsky 2015). To identify the relationship between OsRopGEF7B and RAC/ROPS, we carried out the bimolecular fluorescence complement (BiFC) analysis in the plant cells. When BiFC constructs of OsRopGEF7B in combination with seven individual OsRACs were cotransformed into rice protoplasts, BiFC-generated apparent yellow fluorescent protein (YFP) signal in the plasma membrane was observed (Fig. 6b-d, f-h) except for OsRAC4 which only shows very weak YFP signal (Fig. 6e). The controls displayed only background signal (Fig. 6a), indicating that OsRopGEF7B interacted with 

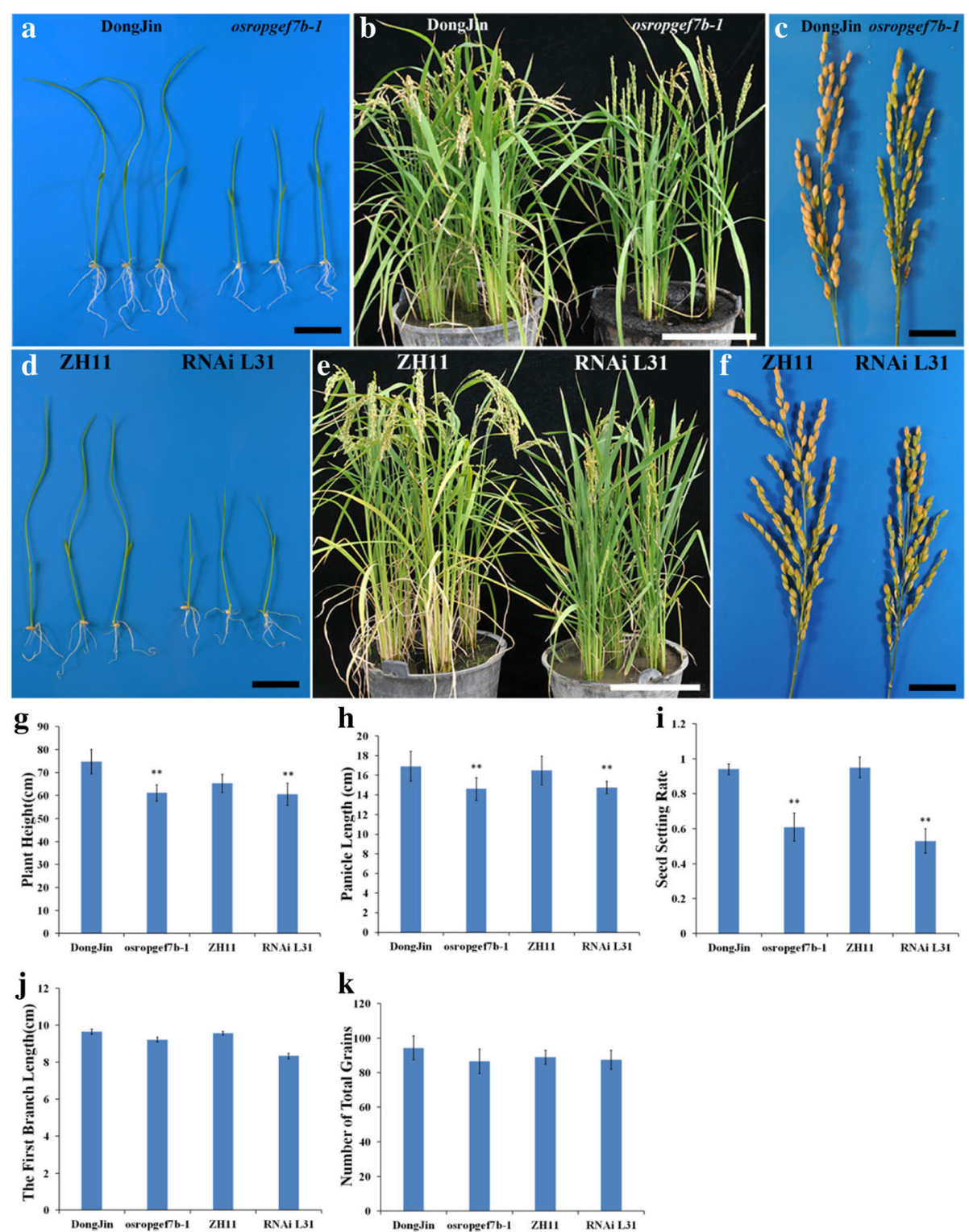

Fig. 5 OsRopGEFTB affects agronomic traits in rice. (A-C) Phenotypic differences between osropgef7b-1 and wild type. a 7-d-old seedling stage. b Mature stage. c Panicles. $\mathbf{d}$-f) Phenotypes of RNAi L31 compared with wild type. $\mathbf{d}$ 7-d-old seedling stage. e Mature stage. $\mathbf{f}$ Panicles. $\mathbf{g}-\mathbf{k}$ Main agronomic traits were influenced by the knock-out or knock-down of OsRopGEF7B in osropgef7b-1 and RNAi L31 compared with wild type. $\mathbf{g}$ Plant height. $\mathbf{h}$ Panicle length. i Seed setting rate. $\mathbf{j}$ Length of first branch. $\mathbf{k}$ Number of total grains per panicle. Data were presented as mean values of three biological replicates with SD, $n=60$ to 80 . Scale bars: (a, c, d, f) $5 \mathrm{~cm}$; (b, e) $30 \mathrm{~cm}$

all of seven OsRACs in plasma membrane in rice protoplasts. To further identify the interactions between OsRopGEF7B and OsRACs, we performed the yeast two-hybrid $(\mathrm{Y} 2 \mathrm{H})$ assay. The results suggested that OsRopGEF7B strongly interacts with six OsRACs but only weakly with OsRAC4 (yeast growth seen only after seven days, Additional file 1: Figure S6B) on quadruple dropout medium supplemented with $3 \mathrm{mM} 3$-AT, compared to both positive and negative controls (Fig. 7; Additional file 1: Figure S6B). The BiFC and $\mathrm{Y} 2 \mathrm{H}$ analyses suggested that OsRopGEF7B strongly interacts with six OsRACs but only very weakly with OsRAC4. Taken together, our data suggest that OsRopGEF7B may activate OsRACs through direct interaction in the signaling pathway, to regulate floral organs development in rice.

\section{Discussion}

The roles of RopGEFs in regulating plant growth and development in rice were rarely reported. Here, we provided experimental evidence that OsRopGEF7B has functions during the vegetative growth and reproductive 


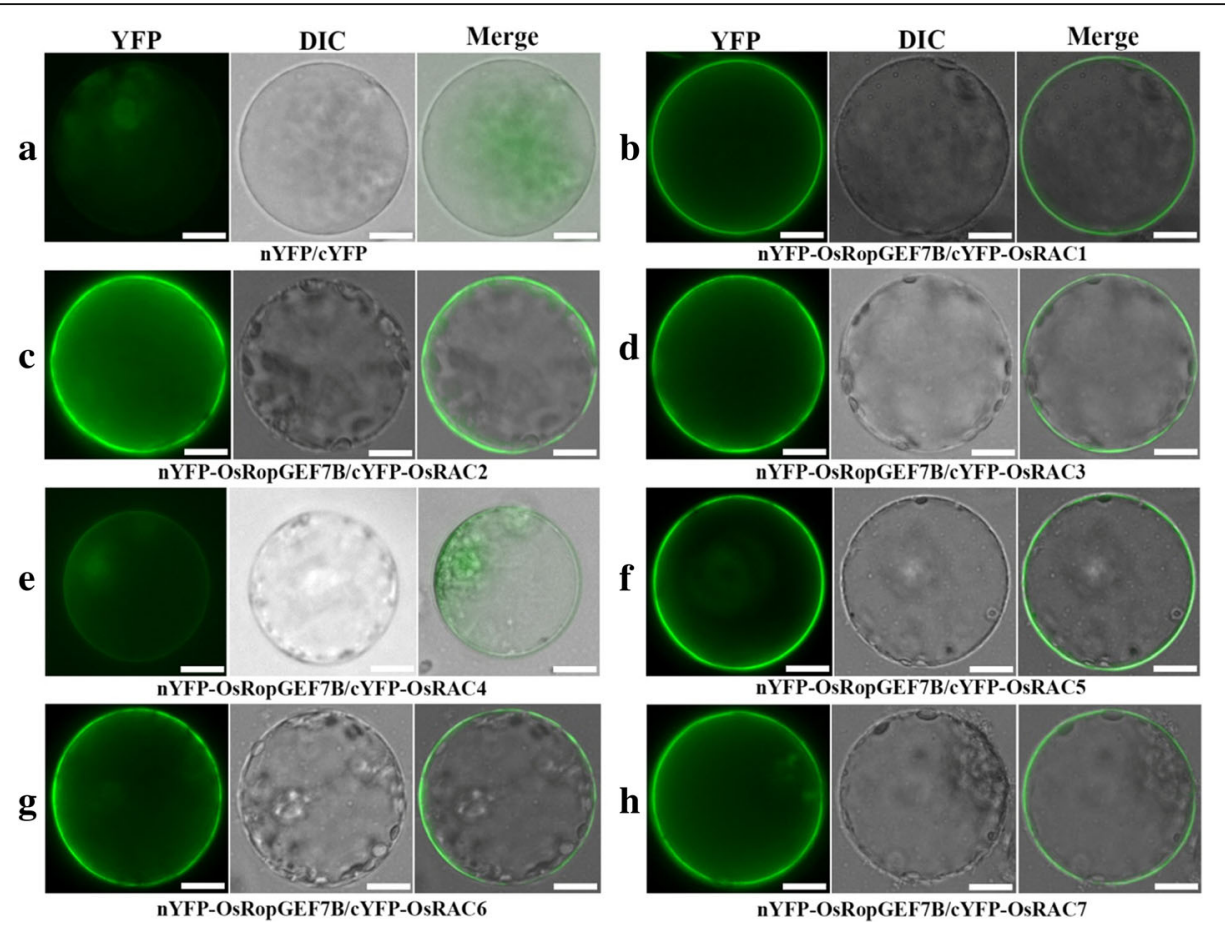

Fig. 6 OsRopGEF7B interacts with OsRACs in rice protoplasts. Rice protoplasts were co-transfected with (a) N-terminal YFP and C-terminal YFP, (b) nYFP-OsRopGEFTB and CYFP-OsRAC1, (c) nYFP-OsRopGEFTB and CYFP-OSRAC2, (d) nYFP-OsRopGEFTB and cYFP-OsRAC3, (e) nYFP-OsRopGEFTB and CYFP-OsRAC4, (f) nYFP-OsRopGEF7B and CYFP-OsRAC5, (g) nYFP-OsRopGEF7B and CYFP-OsRAC6, (h) nYFP-OsRopGEF7B and CYFP-OsRAC7. Images were acquired under the YFP and differential interference contrast (DIC) channel, respectively, and then merged. Bars $=10 \mu \mathrm{m}$

development in rice, especially in the processes of floral organ development.

Our data revealed that OsRopGEF7B is predominantly expressed in floral meristem, floral organ primordia, anther, filament and stigma (Fig. 1e-l). The knock-out mutant and knock-down transgenic plants, osropgef $7 b-1$ and RNAi L31, respectively, showed increases in the numbers of the inner floral organs (ovary and stamen) (Table 1; Figs. 2i-q, 4f-j). Specifically, some of the osropgef7b-1(10.13\%) and RNAi L31 (3.95\%) plants have more than one ovary (Table 1; Figs. 2i-q, 4f-j). In the wild type flower, carpel is composed of ovary, style and stigma; the carpel primordium is developed from the floral meristem (Chu et al. 2006). Initiation of the floral meristems is the start of flower development, followed by floral meristem identity specification and maintenance, floral organ primordia initiation, floral organ identity specification, floral stem cell termination, and finally floral organ maturation (Guo et al. 2015). In this multistep process, each of numerous genes is expressed in a spatiotemporally regulated manner. Phenotypes of mutants give clues which role the corresponding gene plays in flower development. The floral organ number1 (fon1) mutant of Arabidopsis shows abnormal flowers from stage 6, after the three-whorl stamen primordia have initiated. The prolonged floral meristem activity continues to produce extra stamen and carpel primordia, resulting in generating additional stamens and carpels (Huang and Ma, 1997). The fon 1 mutant of rice also exhibits an enlarged floral meristem and subsequently increased the number of all floral organs (Nagasawa et al., 1996; Suzaki et al., 2004). FON4 of rice plays a more important role on the carpel development than on the outer whorls; the fon41 displayed an increase in the number of carpel that results from the extra carpel primordia in the enlarged floral meristem; almost all fon4-1 and fon4-2 flowers and most of fon4-3 flowers contain more than one carpel (Chu et. 2006). Thus, phenotypic similarities between osropgef7b-1 (and RNAi L31) and fon mutants suggest that mutation in OsRopGEF7B might strengthen the activity of flower meristem subsequently producing extra carpel primordia resulting in the generation of additional carpels (as well as additional ovaries). Thus, normal expression of OsRopGEF7B seems to be required for restricting the numbers of inner floral organs. To understand the mechanism underlying this phenotype, the detailed morphological analysis of the floral meristem in osropgef7b-1 and RNAi L31 plants compared to wild type is needed in the future study.

Previously, our group reported that Arabidopsis RopGEF7, grouped into the same clade with OsRopGEF7B (Additional file 1: Figure S7), maintains the stem cell 

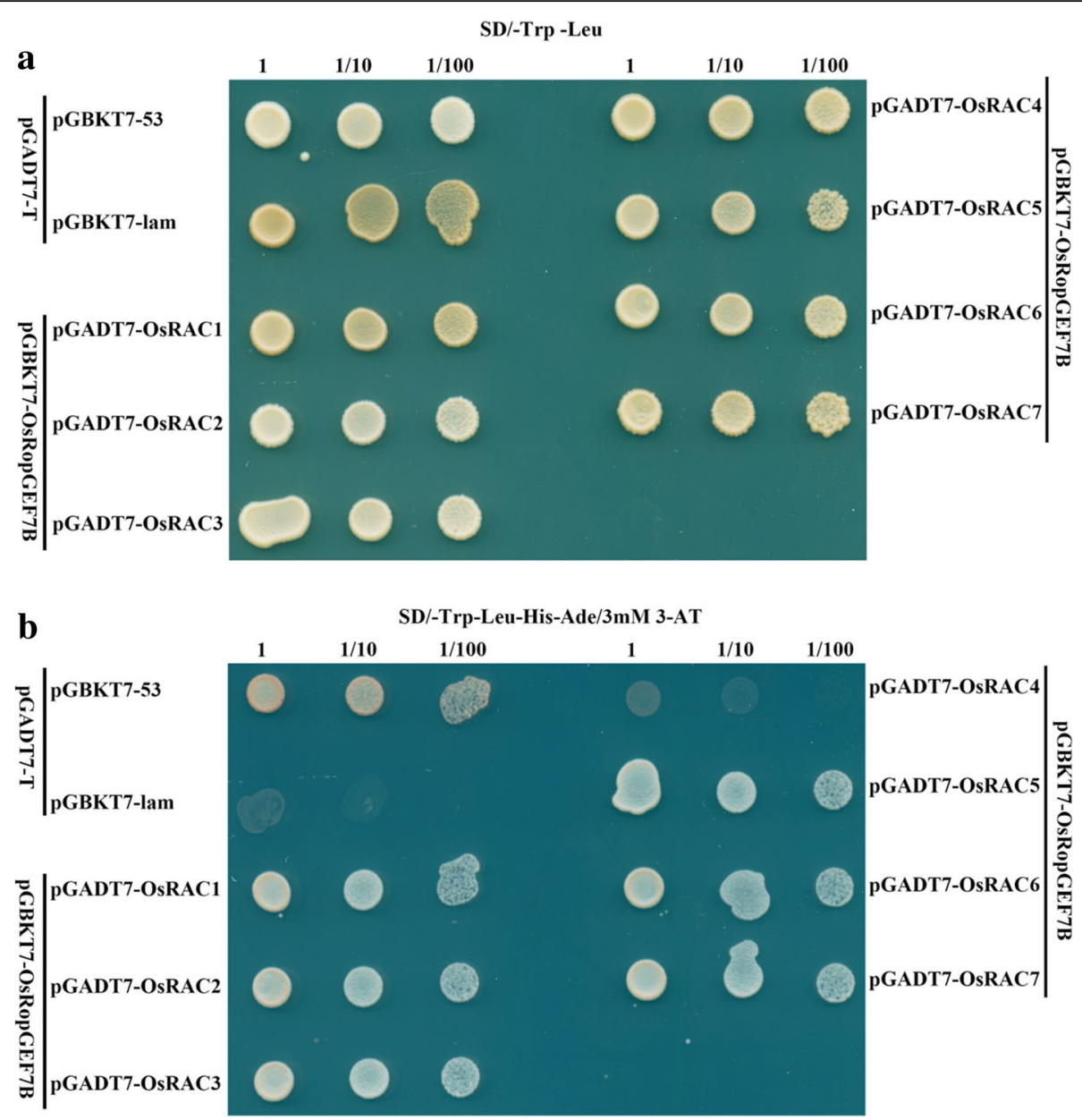

Fig. 7 Interactions between OsRopGEF7B and OsRACs in the $\mathrm{Y} 2 \mathrm{H}$ assay. The transformants were plated onto (a) double dropout medium (SD/-Trp/-Leu) and onto (b) quadruple dropout medium (SD/-Trp/-Leu/-His/-Ade) supplemented with 3-AT. The pairs of pGBKT7-53 / pGADT7-T and PGBKT7-Lam / pGADT7-T were used as positive and negative controls, respectively. The growth of co-transformed yeast cells in the quadruple dropout medium supplemented with 3-AT is indicative of protein-protein interaction in yeast

niche by auxin-dependent PLT pathway. Knock-down of AtRopGEF7 by RNA interference technique indicated that AtRopGEF7 can regulate the auxin efflux transporter PIN1, PIN3 and PIN7 accumulation and thereby affects polar auxin transport that affects root development (Chen et al. 2011; Huang et al. 2014). Additionally, auxin plays vital roles in determining floral organ development. Plants harboring mutations in PIN1 often show strong defects in inflorescence with naked stems, often produce only a few abnormal flowers. pin1 mutant flowers exhibit variable petals number, fused floral organs, and expansion of the stylar and stigmatic regions of the gynoecium (Okadala et al. 1991; Cheng and Zhao 2007; Yamaguchi et al. 2013; Holt et al. 2014). Similarly, osropgef7b-1 and RNAi L31 exhibited fused ovaries (Table 1; Figs. 2i-q, 4f-j), and osropgef7b-1 showed three glumes (Fig. 2h). Though our results indicated that the transcripts of all four OsPIN1 members are maintained at the same levels in osropgef $7 b-1$ and RNAi L31 as those in the wild type (Additional file 1: Figure S5C, D), we could not exclude the possibility that OsRopGEF7B might regulate PIN1 at the posttranscriptional level.

In Arabidopsis, overexpression of RopGEF1 and RopGRF12 affects pollen tube growth (Gu et al. 2006; Zhang and McCormick 2007). Meanwhile, the microarray data from the RiceXPro online database (http:// ricexpro.dna.affrc.go.jp/category-select.php) showed that nine out of eleven rice RopGEFs were highly expressed in anther. The OsRopGEF7B ${ }_{p r o}$ :GUS analysis results indicate that OsRopGEF7B is strongly expressed in anther (Fig. 1i-l). However, our data indicated that knock-out or -down of OsRopGEF7B does not affect pollen development (Additional file 1: Figure S3) and pollen tube elongation (Additional file 1: Figure S4), which might be explained by the functional redundancy of OsRopGEF members. Interestingly, we also found that knock-out or -down of OsRopGEF7B markedly influenced plant height, panicle length, and seed setting rates in 
osropgef7b-1 and RNAi L31 in comparison with wild type (Fig. 5a-i). Taken together, these results suggest that OsRopGEF7B influences rice seed setting partly through mediating floral organ development.

The X-ray structure analysis provided that the catalytic PRONE domain of AtRopGEF8 is found in a ternary complex with Rop4 and GDP (Thomas et al., 2007) and immunity studies have provided evidence that PRONE-type OsRacGEFs may be involved in disease responses through activation of OsRAC1 in rice (Kawasaki et al. 2009; Kawano et al., 2010), and OsRopGEF10 regulates small papillae development through activating OsRAC1 (Yoo et al. 2011). Additionally, yeast two-hybrid assays displayed that OsRAC1 interacts with OsRacGEF1 and OsRacGEF2 via their PRONE domain (Akamatsu et al. 2013, 2015), and the CEBiP/CERK1-OsRacGEF-OsRac1 module plays a major role for early signaling in rice chitin-triggered immunity (Akamatsu et al. 2013). The above-mentioned evidence suggested that OsRopGEFs act at the upstream of OsRACs. Given the interactions between RopGEFs and RACs during various biological processes in the plant cells, we further investigated the relationship between RopGEFs and RACs in rice protoplasts and yeast cells. Expectedly, the BiFC analysis indicated that OsRopGEF7B interacts with all OsRACs at plasma membrane but OsRAC4 which only shows very weak interaction (Figure 6), and the $\mathrm{Y} 2 \mathrm{H}$ assay confirmed the results of the BiFC analysis (Figure 7; Additional file 1: Figure S6B). Therefore, it is speculated that RopGEF7B might interact with OsRACs in plant cells. Identifying the exact cooperative roles of OsRopGEF7B and OsRACs will help to further elucidate how RopGEF7B regulates rice floral development.

\section{Conclusions}

OsRopGEF7B is predominantly expressed in floral organs, especially in meristem, floral organ primordia, anther, filament and stigma. osropgef7b-1 mutant and RNAi L31 transgenic plants, exhibited increase in the numbers of the inner floral organs (ovary and stamen), generating decrement in seed setting. Meanwhile, OsRopGEF7B strongly interacted with OsRACs except the OsRAC4 which only shows very weak interaction. It suggested that OsRopGEF7B mediates the development of floral organs via activating OsRACs in rice. Understanding the function of OsRopGEF7B gives new clues to further elucidate the development of rice floral organs and has possible application in genetically modified crops.

\section{Methods}

Plant Materials and Growth Conditions

Rice plants (Oryza sativa cv. Zhonghua11 (ZH11), DongJin, RNAi lines, and osropgef $7 b-1$ ) were grown under normal field conditions in the rice growing season at the South China Agricultural University, Guangzhou, or grown in a growth chamber under 14-h-light long-day conditions at $28{ }^{\circ} \mathrm{C}$ day/night cycles.

\section{Chimeric Gene Construction and Plant Transformation}

The OsRopGEF7B $B_{\text {pro }}$ :GUS construct was generated by inserting a $3024 \mathrm{bp}$ OsRopGEF7B promoter sequence into pCAMBIA 1305.1 at PstI and NcoI sites. To construct the RNAi vector, a 327 bp fragment of OsRop$G E F 7 B$ was amplified from the cDNA pool using the primer set OsRopGEF7B-RNAi-F and OsRopGEF7B-R$N A i-\mathrm{R}$, then inserted into the BamHI and HindIII sites (for forward insertion) and the MluI and Pst $\mathrm{I}$ sites (for reverse insertion) of the pYLRNAi.5 vector with an Ubiquitin promoter provided by Dr. Yao-Guang Liu (Luo et al. 2013). These binary constructs were introduced into Agrobacterium tumefaciens strain EHA105 and were used for rice transformation following a previously described protocol (Li et al. 2011; Liu et al. 2014). Primers for generating these constructs were listed in Additional file 2: Table S1.

\section{RNA Isolation and qRT-PCR Analyses}

For analyzing gene expression, total RNA was extracted using the RNeasy plant mini kit (Qiagen). Genomic DNA contamination was removed by RNase-free DNase I treatment. First-strand cDNA was synthesized with Primescript cDNA synthesis kit (TaKaRa) and oligo (dT) primers. qRT-PCR analysis was performed using the synthesized cDNAs and the primers listed in Additional file 2: Table S1. Constitutively expressed OsActin1 (accession No. LOC_Os03g50885) was used as an internal control to which the level of OsRopGEF7B in different tissues was normalized. Data are presented as averages with SD from at least three biological replicates.

\section{Paraffin Section and Microscopy}

Rice spikelets were fixed in a fixative solution (50\% ethanol, 10\% glacial acetic acid and 3.7\% formaldehyde) overnight at $4{ }^{\circ} \mathrm{C}$. After dehydration with ethanol in a series of concentrations and infiltration with xylene, the materials were embedded in paraffin (Sigma-Aldrich) as described before (Zhang et al. 2010). Sections of $8 \mu \mathrm{m}$ in thickness were cut with a microtome and stained with $0.05 \%$ toluidine blue. Samples were observed using Nomarski optics on an Olympus BX51 microscope connected to a Ritiga 2000R digital camera.

\section{Pollen in Vivo Germination and Elongation Analyses}

To examine the pollen grains, rice mature flowers 1 day prior to anthesis were collected and fixed in 70\% $(\mathrm{v} / \mathrm{v})$ ethanol and then the pollen grains were stained with $\mathrm{I}_{2}$-KI staining as described by Huang et al. (2014). The 
pollen grains were counted under a bright field microscope (Olympus BX51) and densely stained pollen grains were counted as fertile pollen grains. For 4',6-diamidino-2-phenylindole (DAPI) staining, pollen grains from the dehisced anthers were fixed in 3:1 ethanol:acetic acid (EAA) solution for $1 \mathrm{~h}$, then dehydrated and stained with DAPI. The stained pollen grains were observed using a microscope under UV light (Leica DM2500).

To analyze the pollen in vivo germination, rice flowers were emasculated and artificially pollinated. After $30 \mathrm{~min}$, the pistils were collected and directly stained with aniline blue on a glass slide for 2-3 min before observation by fluorescence microscopy (Olympus BX51) as described by Chhun et al. (2007). The pollen tube growth of germinated pollen attached to stigmas was visualized by fluorescence microscopy with CFP channel.

To assay pollen in vivo elongation, at $30 \mathrm{~min}, 1 \mathrm{~h}$, and $2 \mathrm{~h}$ time points following artificial pollination, rice pistils were collected and fixed in EAA solution for $30 \mathrm{~min}$ and then softened in $1 \mathrm{~N} \mathrm{KOH}$ for $30 \mathrm{~min}$ at $55{ }^{\circ} \mathrm{C}$. Next, the pistils were washed with PBS buffer for $2 \mathrm{~h}$ at room temperature. The samples were observed by fluorescence microscopy (Olympus BX51) with CFP channel. Pollen elongation can be seen in a time-dependent manner (Chhun et al. 2007).

\section{Bimolecular Fluorescence Complement (BiFC) Assay in Rice Protoplasts}

To generate the fusion proteins of nYFP-OsRopGEF7B and cYFP-OsRACs, the coding sequences of OsRopGEF7B and OsRACs were inserted into the EcoRI and SalI sites of pSAT6-nEYFP-C1 and pSAT6-cEYFP-C1 vectors (Citovsky et al. 2006), respectively. Both plasmids

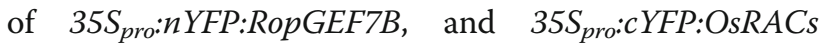
were cotransformed into rice protoplasts for BiFC assays. Protoplast isolation and transfection were performed as described by Tao et al. (2005). YFP was visualized by an Olympus BX51 microscope using YFP filter sets: the excitation and emission filters Ex490 to $510 \mathrm{~nm} / \mathrm{DM} 515 \mathrm{~nm} / \mathrm{BA} 520$ to $550 \mathrm{~nm}$ (Tao et al. 2005). Primers used for constructing the above-mentioned plasmids were listed in Additional file 2: Table S1 online.

\section{Yeast Two-Hybrid Assay}

The full-length coding sequences of OsRopGEF7B and OsRACs were amplified and cloned into pGBKT7 (BD, Clontech) and pGADT7 (AD, Clontech) vectors, respectively. The primers used to generate the $\mathrm{Y} 2 \mathrm{H}$ constructs were listed in Additional file 1: Table S1. The constructs were co-transformed by pairs into the yeast stain AH109, and the transformants were selected on synthetic dextrose (SD) medium lacking tryptophan and leucine (SD/-Trp/-Leu) following incubation at $28{ }^{\circ} \mathrm{C}$ for 3 4 days according to the Yeast Protocol Handbook
(Cat. No. 630412, Clontech, Japan). Single co-transformed yeast clones of $\mathrm{SD} /-\mathrm{Trp} /$-Leu plates were transferred to SD medium lacking tryptophan, leucine, histidine and adenine (SD/-Trp/-Leu/-His/-Ade) in 10-fold serial dilutions to identify the protein-protein interactions. The positive (pGBKT7-53 / pGADT7-T) and negative (pGBKT7-Lam / pGADT7-T) controls were used. After confirmation that neither autoactivation nor toxicity exists in those yeast cells co-transformed with pGBKT7-OsRopGEF7B and the null pGADT7 vector as well as pGADT7-OsRACs and the null pGBKT7 vector, respectively, the $\mathrm{Y} 2 \mathrm{H}$ screening was performed according the procedure in the Yeast Protocol Handbook. The potential protein-protein interactions were confirmed by growing the yeast cells in $\mathrm{SD} /-\operatorname{Trp} /-\mathrm{Leu} /-\mathrm{His} /-$ Ade/ 3-AT plates supplemented with $3 \mathrm{mM}$ 3-AT (3-amino-1,2,4-triazole, Sigma-Aldrich).

\section{Statistics}

All the data represented the average with the standard deviation of the average (SD) from three biological experiments. Significant difference was determined by paired two-tailed Student's $t$-tests. $P<0.05$ was considered significant.

\section{Additional files}

Additional file 1: Figure S1. Relative expression levels of OsRopGEF7B in various tissues of rice at vegetative and reproductive stages. Figure S2. Relative expression levels of OsRopGEF7B in OsRopGEF7B-RNAi lines. Figure S3. OsRopGEF7B does not affect pollen development. Figure S4. In vivo pollen germination and PT elongation. Figure S5. Relative expression levels of a subset of genes associated with floral development in osropgef7b-1 mutant and RNAi L31 line at both seedling and floral stages. Figure S6. Interactions between OsRopGEF7B and OsRACs in the $\mathrm{Y} 2 \mathrm{H}$ assay after seven days of growth. Figure S7. Phylogenetic relationships between OsRopGEFs and AtRopGEFs. (PPTX 4833 kb)

Additional file 2: Table S1. List of primers used in this study. (DOCX $20 \mathrm{~kb}$ )

\section{Acknowledgements}

We thank Yaoguang Liu and Hongquan Yang for providing RNAi and BiFC vectors. We thank Junli Huang for comments on the manuscript and Dongyu Jia for helpful discussion on this work.

\section{Funding}

This research was supported by grants from the National Natural Science Foundation of China (91535101 and 31600217).

\section{Availability of Data and Materials}

All relevant data are provided as figures within the paper.

\section{Authors' Contributions}

$\mathrm{J} H$ performed the experiments; $H \mathrm{~L}$ and $\mathrm{Y} L$ provide technical assistant to J $\mathrm{H} ; \mathrm{T}$ B discussed and revised the manuscript. L T designed experiments and modified the manuscript; $T L$ designed and performed the experiments and wrote the manuscript. All authors read and approved the final manuscript.

\section{Authors' Information}

${ }^{1}$ State Key Laboratory for Conservation and Utilization of Subtropical Agrobioresources, South China Agricultural University, Guangzhou 510,642, China. ${ }^{2}$ Guangdong Provincial Key Laboratory of Protein Function and Regulation in 
Agricultural Organisms, College of Life Sciences, South China Agricultural University, Guangzhou 510,642, China. ${ }^{3}$ Senckenberg Biodiversity and Climate Research Center, Georg-Voigt-Str. 14-16, D-60325 Frankfurt am Main, Germany.

\section{Ethics Approval and Consent to Participate}

There has no ethics problems involved in this article.

\section{Consent for Publication}

These co-authors involved in the paper all consent to publish this article on Rice.

\section{Competing Interests}

The authors declare that they have no competing interests.

\section{Publisher's Note}

Springer Nature remains neutral with regard to jurisdictional claims in published maps and institutional affiliations.

\section{Author details Main, Germany. \\ Received: 16 March 2018 Accepted: 10 July 2018 \\ Published online: 30 July 2018}

'State Key Laboratory for Conservation and Utilization of Subtropical Agro-bioresources, South China Agricultural University, Guangzhou 510642, China. ${ }^{2}$ Guangdong Provincial Key Laboratory of Protein Function and Regulation in Agricultural Organisms, College of Life Sciences, South China Agricultural University, Guangzhou 510642, China. ${ }^{3}$ Senckenberg Biodiversity and Climate Research Center, Georg-Voigt-Str. 14-16, D-60325 Frankfurt am

\section{References}

Akamatsu A, Uno K, Kato M, Wong HL, Shimamoto K, Kawano Y (2015) New insights into the dimerization of small GTPase Rac/ROP guanine nucleotide exchange factors in rice. Plant Signal Behav 10:e1044702

Akamatsu A, Wong HL, Fujiwara M, Okuda J, Nishide K, Uno K, Imai K, Umemura K, Kawasaki T, Kawano Y, Shimamoto K (2013) An OsCEBiP/ OsCERK1-OsRacGEF1-OsRac1 module is an essential early component of chitin-induced rice immunity. Cell Host Microbe 13:465-476

Berken A, Thomas C, Wittinghofer A (2005) A new family of RhoGEFs activates the Rop molecular switch in plants. Nature 436:1176-1180

Bloch D, Yalovsky S (2013) Cell polarity signaling. Curr Opin Plant Biol 16:734-742

Bourne HR, Sanders DA, McCormick F (1990) The GTPase superfamily: a conserved switch for diverse cell functions. Nature 348:125-132

Bourne HR, Sanders DA, McCormick F (1991) The GTPase superfamily: conserved structure and molecular mechanism. Nature 349:117-127

Chang F, Gu Y, Ma H, Yang Z (2013) AtPRK2 promotes ROP1 activation via RopGEFs in the control of polarized pollen tube growth. Mol Plant 6:1187-1201

Chen M, Liu H, Kong J, Yang Y, Zhang N, Li R, Yue J, Huang J, Li C, Cheung AY, Tao LZ (2011) RopGEF7 regulates PLETHORA-dependent maintenance of the root stem cell niche in Arabidopsis. Plant Cell 23:2880-2894

Cheng Y, Zhao Y (2007) A role for auxin in flower development. J Integ Plant Biol 49:99-104

Cherfils J, Chardin P (1999) GEFs: structural basis for their activation of small GTP-binding proteins. Trends Biochem Sci 24:306-311

Chhun T, Aya K, Asano K, Yamamoto E, Morinaka Y, Watanabe M, Kitano H, Ashikari M, Matsuoka M, Ueguchi-Tanaka M (2007) Gibberellin regulates pollen viability and pollen tube growth in rice. Plant Cell 19:3876-3888

Chu H, Qian Q, Liang W, Yin C, Tan H, Yao X, Yuan Z, Yang J, Huang H, Luo D, Ma H, Zhang D (2006) The floral organ number4 gene encoding a putative ortholog of Arabidopsis CLAVATA3 regulates apical meristem size in rice. Plant Physiol 142:1039-1052

Citovsky V, Lee LY, Vyas S, Glick E, Chen MH, Vainstein A, Gafni Y, Gelvin SB, Tzfira $T$ (2006) Subcellular localization of interacting proteins by bimolecular fluorescence complementation in planta. J Mol Biol 362:1120-1131

Craddock C, Lavagi I, Yang Z (2012) New insights into rho signaling from plant ROP/Rac GTPases. Trends Cell Biol 22:492-501

Gu Y, Li S, Lord EM, Yang Z (2006) Members of a novel class of Arabidopsis rho guanine nucleotide exchange factors control rho GTPase-dependent polar growth. Plant Cell 18:366-381
Guo S, Sun B, Looi L-S, Xu Y, Gan E-S, Huang J, Ito T (2015) Co-ordination of flower development through epigenetic regulation in two model species: Rice and Arabidopsis. Plant Cell Physiol 56:830-842

Holt AL, van Haperen JM, Groot EP, Laux T (2014) Signaling in shoot and flower meristems of Arabidopsis thaliana. Curr Opin Plant Biol 17:96-102

Huang H, Ma H (1997) FON1, an Arabidopsis gene that terminates floral meristem activity and controls flower organ number. Plant Cell 9:115-134

Huang JB, Liu H, Chen M, Li X, Wang M, Yang Y, Wang C, Huang J, Liu G, Liu Y, Xu J, Cheung AY, Tao LZ (2014) ROP3 GTPase contributes to polar auxin transport and auxin responses and is important for embryogenesis and seedling growth in Arabidopsis. Plant Cell 26:3501-3518

Ikeda K, Sunohara H, Nagato Y (2004) Developmental course of inflorescence and spikelet in rice. Breed Sci 54(2):147-156

Kawano Y, Chen L, Shimamoto K (2010) The function of Rac small GTPase and associated proteins in Rice innate immunity. Rice 3:112-121

Kawasaki T, Imai K, Wong HL, Kawano Y, Nishide K, Okuda J, Shimamoto K (2009) Rice guanine nucleotide exchange factors for small GTPase OsRac1 involved in innate immunity of rice. Advances in genetics, genomics and control of rice Blast disease. Springer, Netherlands, pp 179-184

Li J, Jiang D, Zhou H, Li F, Yang J, Hong L, Fu X, Li Z, Liu Z, Li J, Zhuang C (2011) Expression of RNA-interference/antisense transgenes by the cognate promoters of target genes is a better gene-silencing strategy to study gene functions in rice. PLoS One 6:e17444

Li Z, Liu D (2012) ROPGEF1 and ROPGEF4 are functional regulators of ROP11 GTPase in ABA-mediated stomatal closure in Arabidopsis. FEBS Lett 586:1253-1258

Li Z, Waadt R, Schroeder JI (2016) Release of GTP exchange factor mediated down-regulation of abscisic acid signal transduction through ABA-induced rapid degradation of RopGEFs. PLoS Biol 14:e1002461

Liu T, Kim DW, Niitsu M, Maeda S, Watanabe M, Kamio Y, Berberich T, Kusano T (2014) Polyamine oxidase 7 is a terminal catabolism-type enzyme in Oryza sativa and is specifically expressed in anthers. Plant Cell Physiol 55:1110-1122

Liu Y, Dong Q, Kita D, Huang JB, Liu G, Wu X, Zhu X, Cheung AY, Wu HM, Tao LZ (2017) RopGEF1 plays a critical role in polar auxin transport in early development. Plant Physiol 175:157-171

Luo D, Xu H, Liu Z, Guo J, Li H, Chen L, Fang C, Zhang Q, Bai M, Yao N, Wu H, Wu H, Ji C, Zheng H, Chen Y, Ye S, Li X, Zhao X, Li R, Liu YG (2013) A detrimental mitochondrial-nuclear interaction causes cytoplasmic male sterility in rice. Nat Genet 45:573-577

Nagasawa N, Miyoshi M, Kitano H, Satoh H, Nagato Y (1996) Mutations associated with floral organ number in rice. Planta 198:627-633

Nemhauser JL, Feldman LJ, Zambryski PC (2000) Auxin and ETTIN in Arabidopsis gynoecium morphogenesis. Development 127:3877-3888

Nibau C, Tao L, Levasseur K, Wu HM, Cheung AY (2013) The Arabidopsis small GTPase AtRAC7/ROP9 is a modulator of auxin and abscisic acid signalling. J Exp Bot 64:3425-3437

Okadala K, Ueda J, Komaki MK, Bell CJ, Shimura Y (1991) Requirement of the auxin polar transport system in early stages of Arabídopsis floral bud formation. Plant Cell 3:677-684

Prasad K, Parameswaran S, Vijayraghavan U (2005) OsMADS1, a rice MADS-box factor, controls differentiation of specific cell types in the lemma and Palea and is an early-acting regulator of inner floral organs. Plant J 43:915-928

Sessions A, Nemhauser JL, McColl A, Roe JL, Feldmann KA, Zambryski PC (1997) ETTIN patterns the Arabidopsis floral meristem and reproductive organs. Development 124:4481-4491

Shichrur K, Yalovsky S (2006) Turning ON the switch--RhoGEFs in plants. Trends Plant Sci 11:57-59

Suzaki T, Sato M, Ashikari M, Miyoshi M, Nagato Y, Hirano HY (2004) The gene FLORAL ORGAN NUMBER1 regulates floral meristem size in rice and encodes a leucine-rich repeat receptor kinase orthologous to Arabidopsis CLAVATA1. Development 131:5649-5657

Tao LZ, Cheung AY, Nibau C, Wu HM (2005) RAC GTPases in tobacco and Arabidopsis mediate auxin-induced formation of proteolytically active nuclear protein bodies that contain AUX/IAA proteins. Plant Cell 17: 2369-2383

Teo ZW, Song S, Wang YQ, Liu J, Yu H (2014) New insights into the regulation of inflorescence architecture. Trends Plant Sci 19:158-165

Thomas C, Fricke I, Scrima A, Berken A, Wittinghofer A (2007) Structural evidence for a common intermediate in small $\mathrm{G}$ protein-GEF reactions. Mol Cell 25:141-149 
Wu HM, Hazak O, Cheung AY, Yalovsky S (2011) RAC/ROP GTPases and auxin signaling. Plant Cell 23:1208-1218

Xin Z, Zhao Y, Zheng ZL (2005) Transcriptome analysis reveals specific modulation of abscisic acid signaling by ROP10 small GTPase in Arabidopsis. Plant Physiol 139:1350-1365

Yadav SR, Khanday I, Majhi BB, Veluthambi K, Vijayraghavan U (2011) Auxinresponsive OsMGH3, a common downstream target of OsMADS1 and OsMADS6, controls rice floret fertility. Plant Cell Physiol 52:2123-2135

Yalovsky S (2015) Protein lipid modifications and the regulation of ROP GTPase function. J Exp Bot 66:1617-1624

Yalovsky S, Bloch D, Sorek N, Kost B (2008) Regulation of membrane trafficking, cytoskeleton dynamics, and cell polarity by ROP/RAC GTPases. Plant Physiol 147: 1527-1543

Yamaguchi N, Wu MF, Winter CM, Berns MC, Nole-Wilson S, Yamaguchi A, Coupland G, Krizek BA, Wagner D (2013) A molecular framework for auxinmediated initiation of flower primordia. Dev Cell 24:271-282

Yang Z, Fu Y (2007) ROP/RAC GTPase signaling. Curr Opin Plant Biol 10:490-494

Yoo JH, Park JH, Cho SH, Yoo SC, Li J, Zhang H, Kim KS, Koh HJ, Paek NC (2011) The rice bright green leaf ( $\mathrm{bg} /$ ) locus encodes OsRopGEF 10, which activates the development of small cuticular papillae on leaf surfaces. Plant Mol Biol 77: $631-641$

Zhang J, Nallamilli BR, Mujahid H, Peng Z (2010) OsMADS6 plays an essential role in endosperm nutrient accumulation and is subject to epigenetic regulation in rice (Oryza sativa). Plant J 64:604-617

Zhang Y, McCormick S (2007) A distinct mechanism regulating a pollen-specific guanine nucleotide exchange factor for the small GTPase Rop in Arabidopsis thaliana. Proc Natl Acad Sci U S A 104:18830-18835

\section{Submit your manuscript to a SpringerOpen ${ }^{\circ}$ journal and benefit from:}

- Convenient online submission

- Rigorous peer review

- Open access: articles freely available online

- High visibility within the field

- Retaining the copyright to your article

Submit your next manuscript at $\boldsymbol{\nabla}$ springeropen.com 